Int. J. Morphol.,

33(2):433-435, 2015.

\title{
Segmento II del Hígado ¿Segmento Lateral Izquierdo o Segmento Posterior Lateral Izquierdo?
}

\author{
Segment II of the Liver, Left Lateral Segment or Left Lateral Posterior Segment?
}

\author{
Eugenia Carvallo $^{* * * *}$; Pamela Carvallo ${ }^{* *, * * *}$; Rafael Coello ${ }^{* * * * * * *}$; Vásquez, B. ${ }^{* * * * *}$
}

CARVallo E.; CARVAllo, P.; COEllo, R. \& VÁSQUEZ, B. Segmento II del hígado ¿Segmento lateral izquierdo o segmento posterior lateral izquierdo?. Int. J. Morphol., 33(2):433-435, 2015.

RESUMEN: La división del hígado en segmentos se basa en que, a partir del hilio, cada una de las ramas de la vena porta está acompañada por una arteria y un conducto biliar. El conocimiento preciso de las estructuras vasculares intrahepáticas y su distribución en segmentos es importante para planificar técnicas resectivas y efectuarlas con éxito; se ha señalado que es necesario conocer la anatomía biliar de los segmentos del hígado, así como sus variantes, para prevenir trombosis o complicaciones isquémicas del segmento lateral izquierdo y la aparición de fístulas biliares después del trasplante parcial de hígado. En la Terminologia Anatomica aparece mencionado el segmento lateral izquierdo (segmento II). Es nuestro objetivo revisar este término y recomendar la inclusión del adjetivo "posterior," quedando como segmento posterior lateral izquierdo. Este cambio tiene relación con los objetivos básicos que fueron propuestos por el Programa Federativo Internacional de Terminologia Anatomica, organismo encargado de regular la comunicación anatómica y de recibir recomendaciones como la presente, admitiéndose que las incorporaciones o modificaciones terminológicas deben ser claras, armónicas con la región o estructura y con un valor preferiblemente informativo y descriptivo. Las ramas terminales de la vena porta son dos: las venas portas derecha e izquierda. Esta última emite una vena lateral izquierda, cuyo trayecto es horizontal, posterior e izquierdo correspondiente al segmento lateral izquierdo (II). En base a lo anterior, se justificaría denominar e incorporar a Terminologia Anatomica éste segmento como segmento posterior lateral izquierdo.

PALABRAS CLAVE: Segmento II; Segmento lateral izquierdo; Hígado; Terminologia anatomica.

\section{INTRODUCCIÓN}

Desde la segunda mitad del siglo XX la cirugía hepática ha tenido un desarrollo creciente, en parte atribuido a un conocimiento más detallado de la segmentación hepática. Lo anterior dio inicio e hizo posible efectuar resecciones cada vez más regladas, hasta llegar a la práctica de un gran número de hepatectomías para la solución de problemas cada vez más complejos. Así, el conocimiento de la división hepática resulta extremadamente necesario para determinar con exactitud las lesiones de este órgano y orientar en cirugía el abordaje de las mismas (Lo tártaro \& Medan, 2010).

Los Nomina Anatomica de Paris (PNA) fue publicado el año 1955 sin incluir la segmentación hepática. Posteriormente Couinaud, cirujano y anatomista francés en 1957 fue el primero en realizar un detallado estudio de la anatomía intrahepática. Sin embargo, solamente en las últimas ediciones de Nomina Anatomica fue incluida la segmenta-

\begin{tabular}{lc}
\multicolumn{2}{c}{ Segmentación hepática según Couinaud. } \\
\hline \multicolumn{2}{c}{ C. Couinaud } \\
\hline Segmento & Subsegmento \\
Lóbulo caudado & I \\
lateral & II \\
Paramediano & III \\
& IV \\
Paramediano & V \\
& VIII \\
Lateral & VI \\
& VII \\
\hline
\end{tabular}

ción portal hepática, sus partes, divisiones y segmentos. Así aparece mencionado en la parte izquierda del hígado, en su división lateral izquierda, el segmento lateral o segmento II. Posteriormente, en Terminologia Anatomica (2011) se menciona el segmento lateral izquierdo; segmento II. Nuestro objetivo fue revisar este término y referirnos al segmento II como segmento posterior lateral izquierdo. Este cambio tiene relación con los objetivos básicos que fueron propuestos

* Universidad Mayor, Sede Temuco, Chile.

** Doctorado en Ciencia Morfológicas, Universidad de La Frontera, Chile.

*** Universidad Católica de Temuco, Chile.

***** Universidad de Guayaquil, Ecuador.

***** Universidad de Tarapacá, Chile. 
por el Programa Federativo Internacional de Terminologia Anatomica en los cuales se destaca que los términos deben ser, ante todo, simples, con un valor preferiblemente informativo y descriptivo.

\section{FUNDAMENTACIÓN}

De acuerdo a la literatura clásica, la segmentación hepática se basa en las divisiones principales de la arteria hepática, vena porta hepática y conductos hepáticos, formando el pedículo hepático (Moore \& Dalley, 2002). Este pedículo hepático se encuentra rodeado por una cápsula fibrosa perivascular, siendo la vena porta hepática el elemento rector.

Como bien lo menciona Standring (2010), en una visión inferior del hígado, la numeración de los segmentos sigue un espiral de sentido antihorario con centro en la vena porta. Esa numeración comienza en el segmento I y sigue hasta el segmento VI. Los segmentos VII y VIII, más craneales, son numerados en el sentido horario.

A nivel del hilio la vena porta hepática se divide en ramas derecha e izquierda dirigiéndose a las partes derecha e izquierda del hígado, respectivamente. Dentro de cada porción, las ramificaciones primarias de la vena porta hepática tienen la consistencia suficiente para crear segmentos vasculares. El plano horizontal que pasa por el lóbulo derecho y la división lateral del lóbulo izquierdo, más el lóbulo caudado, dividen al hígado en ocho segmentos vasculares. El lóbulo derecho se compone de los segmentos medial anterior derecho; lateral anterior derecho; lateral posterior derecho y medial posterior derecho (segmentos del V a VIII respectivamente), y el lóbulo izquierdo del lóbulo caudado y los segmentos lateral izquierdo; anterior lateral izquierdo y medial anterior izquierdo (segmentos del I al IV respectivamente) de acuerdo a Moore \& Dalley.

Otros autores como Latarjet \& Ruiz Liard (2005), describieron que la rama terminal izquierda a su vez se divide, originando una vena paramediana izquierda correspondiente al los segmentos lateral anterior izquierdo y medial izquierdo (segmentos III y IV) y una vena lateral izquierda cuyo trayecto es horizontal, dirigida hacia atrás y a la izquierda correspondiente al segmento lateral izquierdo (segmento II).

Standring señaló que el segmento II es posterolateral a la fisura izquierda, posee apenas una vaina y drena para la vena hepática izquierda. Sin embargo, cuando se refiere a las divisiones y segmentos del hígado indica que el segmento II corresponde a la división lateral izquierda. De tal manera que, espacialmente, este segmento presenta una posición posterolateral izquierda, concordando con la sugerencia que estamos planteando para este segmento hepático, es decir segmento posterior lateral izquierdo.

El Comité de Terminología de la International Hepato Pancreato Biliary Association (IHPBA), acuñó la llamada "Terminología unificada Brisbane 2000" en la cual menciona que la resección del segmento lateral izquierdo (segmento II), se define desde un punto de vista quirúrgico como sectorectomía lateral izquierda o serectomía posterior izquierda, aludiendo al trayecto de la vena porta.

Estudios de Ribeiro et al. (1998), señalaron la necesidad de conocer la anatomía biliar de los segmentos lateral izquierdo y anterior lateral izquierdo del hígado, así como sus variantes, para prevenir trombosis o complicaciones isquémicas y la aparición de fístulas biliares después del trasplante de hígado parcial.

Por otra parte, es necesario destacar que el lenguage anatómico, constaba a fines del siglo XIX de unos cincuenta mil términos correspondiente a la denominación de aproximadamente seis mil estructuras del cuerpo humano. De esta manera existían alrededor de diez nombres en promedio para cada estructura, lo que significaba una repetición de términos por la utilización de sinónimos y de epónimos. Debido a ello en 1895 se crea Nomina Anatomica entre cuyos objetivos básicos destacan: cada estructura debe ser designada por un solo nombre; cada nombre debe ser corto y simple; las estructuras relacionadas topográficamente por proximidad deben tener los nombres armonizados y los adjetivos calificativos deben ser opuestos. En relación a éste último objetivo, si existe un segmento anterior lateral izquierdo debería describirse un segmento posterior lateral izquierdo en el hígado.

En la actualidad existe Terminologia Anatomica (2011) creado por la Federación Internacional de Asociaciones de Anatomistas (IFAA) y que es elaborada por la Federación Internacional de Programas de Teminología Anatómica (FIPAT). Por otra parte, en América Latina, a través de la Asociación Panamericana de Anatomía, se han estado realizando los Simposios Ibero-latinoamericanos de Terminología Morfológica (SILAT) donde también se está revisando la terminología utilizada en las diversas disciplinas del área morfológica, entre ellas la histológica que como lo señalaron Vásquez \& del Sol (2014) es fundamental en los lenguajes científico y médico, lo que permite una comunicación clara y precisa entre los diversos actores que cultivan las Ciencias Médicas. De esta manera, podemos señalar que el SILAT es una entidad científica-educativa, sin fines de lucro, que se centra en el estudio de los términos médicos en el área de la morfología y que abarca la anatomía, la histología y la embriología. Tiene el objeto de lograr precisión y claridad en las denomi- 
naciones de las estructuras del cuerpo humano (Losardo et al., 2010). Es en esta entidad donde deben ser discutidos los términos anatómicos y por tanto, se debería tratar la nueva denominación para el segmento hepático relacionado con el recorrido posterior de la vena porta hepática en el segmento lateral izquierdo.

Finalmente, hay que señalar que la comprensión actual de la anatomía funcional del hígado, está baseada en la división del hígado realizada por Couinaud (1957). Por otra parte, la comprensión de la anatomía biliar intrahepática, en especial el sistema ductal derecho fue ampliada por Hjortsjo (1948 op cit Standring) y de Healey \& Schroy (1953) que utilizaron el sistema biliar como punto de referencia para la división del hígado. En base a lo anteriormente expuesto, creemos que existe bastante evidencia para denominar e incorporar a Terminologia Anatomica el segmento lateral izquierdo como segmento posterior lateral izquierdo.

CARVallo E.; CARVallo, P.; COEllo, R. \& VÁSQUEZ, B. Segment II of the liver. Left lateral segment or left posterior lateral segment?. Int. J. Morphol., 33(2):433-435, 2015.

SUMMARY: The division of the liver into segments is based on, that originating from the hilum, each of the branches of the portal vein is accompanied by an artery and a bile duct. Accurate knowledge of intrahepatic vascular structures and their distribution is important for planning segment resection techniques and perform them successfully. It should be noted that it is necessary to know the anatomy of the biliary liver segments and its variants, to prevent thrombosis or ischemic complications of the left lateral segment and the appearance of biliary fistulas following partial liver transplant. The left lateral segment (segment II) is mentioned in the Terminologia Anatomica. It is our goal to review this term and recommend inclusion of the word "posterior" remaining as the left posterior lateral segment. This change is related to the basic objectives that were proposed by the Federative International Programme on Anatomical Terminologies, which is responsible for regulating the anatomical communication and get recommendations such as this, admitting that additions or terminological changes should be clear, harmonious with the region or structure and have a preferably informative and descriptive value. The terminal branches of the portal vein are two: the right and left portal veins. The latter emits a left lateral vein, where the trajectory is horizontal, rear and left for the left lateral segment (II). Based on the above information, it would be justified to incorporate this segment as left posterior lateral segment in the Terminologia Anatomica.

KEY WORDS: Segment II; Left lateral segment; Liver; Terminologia Anatomica.

\section{REFERENCIAS BIBLIOGRÁFICAS}

Comité International de la Nomenclature Anatomique. Nomina Anatomica. Paris. Grande Bretagne, Spottiswoode, Ballantyne et Cie, S.A.R.L., Londres, 1955.

Couinaud, C. Le foié: etudes anatomique et chirurgicules. Paris, Masson, 1957.

Federative Comittee of Anatomical Terminology. Terminologia Anatomica. 2. ed. Thieme, 2011.

Healey, J. E. \& Schroy, P. C. Anatomy of the biliary ducts within the human liver; analysis of the prevailing pattern of branchings and the major variations of the biliary ducts. Arch. Surg., 66:599-616, 1953.

Hjortsjo apud Standring, 1948 op cit. Gray's Anatomia. A base anatomica da prática clínica.

Latarjet, M. \& Ruiz Liard, A. Anatomía Humana. $4^{\circ}$ Ed. Buenos Aires, Panamericana, 2005.

Losardo, R. J.; Cruz, G. R.; Rodríguez, T. A.; Prates, J. C. \& Prates, N. E. V. B. Int. J. Morphol., 28(4):1323-6, 2010

Lo Tártaro, M. \& Medan, C. Segmentación hepática y su correlato con imágenes de RMN. Rev. Arg. Anat. Onl., 1(1):17-25, 2010.
Moore, K. L. \& Dailley, K. L. Anatomía, con orientación clínica. 4a. ed. Buenos Aires, Panamericana, 2002.

Ribeiro Jr., M.; Goldenberg, S. \& Prates, J.C. Estudo da anatomia vascular sanguínea e biliar do segmento lateral esquerdo do fígado e sua aplicação cirúrgica. Acta cir. Bras., 13(1):306, 1998.

Standring, S. Gray's Anatomia. A base anatomica da prática clínica. 40 ed. Rio de Janeiro, Elsevier, 2010.

Vásquez, B. \& del Sol, M. La Terminologia Histologica en las Ciencias Médicas. Int. J. Morphol., 32(1):375-80, 2014.

Dirección para correspondencia:

Eugenia Carvallo Semler

Universidad Mayor

Sede Temuco

Avda. Alemania 0281

Temuco - CHILE

Email: eugenia.carvallo@gmail.com

Recibido : 16-10-2014

Aceptado : 22-02-2015 